Musées, Patrimoine et Culture scientifiques et techniques

$128 \mid 2010$

mars - avril 2010

\title{
Le Louvre à Québec, une expérience unique
}

The Louvre to Quebec

\section{Line Ouellet}

URL : http://journals.openedition.org/ocim/165

DOI : 10.4000/ocim.165

ISSN : 2108-646X

Éditeur

OCIM

Édition imprimée

Date de publication : 1 mars 2010

Pagination : 30-35

ISSN : 0994-1908

Référence électronique

Line Ouellet, «Le Louvre à Québec, une expérience unique », La Lettre de I'OCIM [En ligne], 128 | 2010, mis en ligne le 01 mars 2012, consulté le 20 avril 2019. URL : http://journals.openedition.org/ ocim/165; DOI : 10.4000/ocim.165 


\section{Le Louvre à Québec, une expérience unique}

\section{Line Ouellet *}

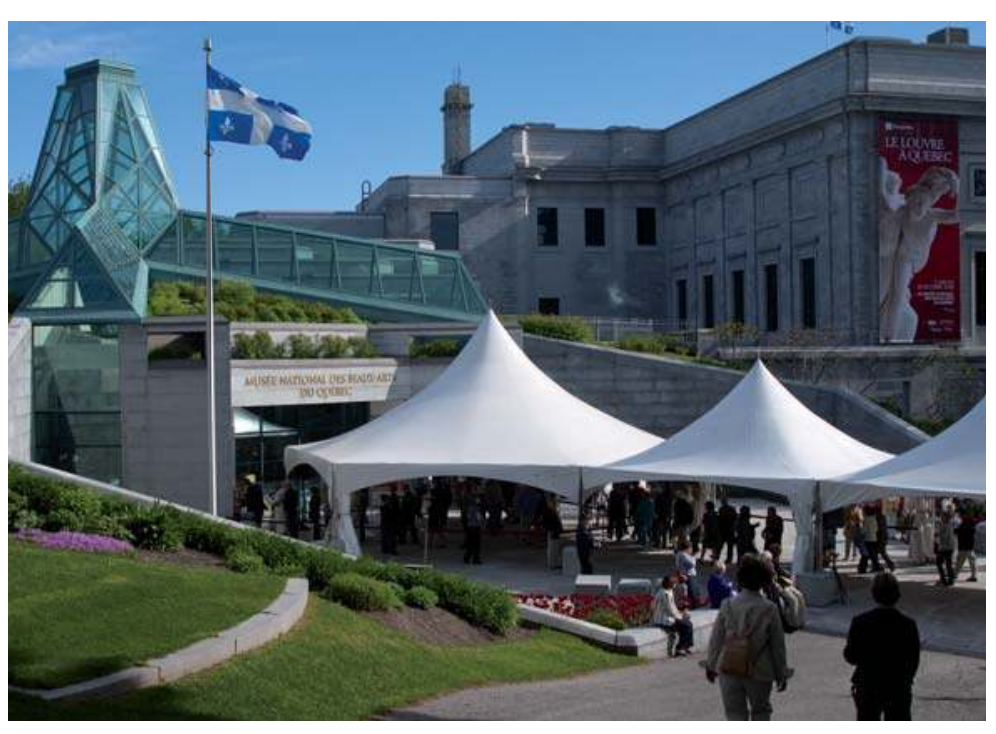

Le musée national des Beaux-Arts du Québec lors de la présentation de l'exposition Le Louvre à Québec. Les arts et la vie à l'été 2008. (c) MNBAQ/Patrick Altman, Jean-Guy Kérouac

* Line Ouellet est directrice des expositions et des publications scientifiques au musée national des Beaux-Arts du Québec line.ouellet@mnba.qc.ca
À travers l'exemple de l'exposition Le Louvre à Québec. Les arts et la vie, issue d'une collaboration entre le musée du Louvre et le musée national des Beaux-Arts du Québec, l'auteur revient sur les éléments d'expérimentation qui ont caractérisé la conception et la réalisation de ce projet et donne les grands principes qui régissent la pratique des expositions de l'établissement québécois.

Le Québec est reconnu pour ses expérimentations en muséologie. Récemment, une collaboration du musée du Louvre avec le musée national des BeauxArts du Québec (MNBAQ) a donné lieu à un mariage inusité entre les pratiques françaises et l'approche québécoise. En effet, à l'occasion du 400e anniversaire de la ville de Québec en 2008, le Louvre a consenti au MNBAQ un prêt exceptionnel de 276 œuvres et, plus encore, a accepté que ses conservateurs travaillent de concert avec des muséologues québécois. Quel était l'objectif de ce projet ? Comment a-t-il été élaboré ? En quoi se distingue-t-il des projets habituels des deux institutions ? Quels en ont été les résultats? Autant de questions que cet article propose de soulever pour mieux éclairer quelques principes et fondements de la pratique des expositions de Beaux-Arts au Québec.

\section{L'objectif et la démarche}

C'est en 2003 qu’Henri Loyrette accepte généreusement de participer au projet. Le directeur du MNBAQ de l'époque, John R. Porter, écrit dans sa 
préface au catalogue : "D'emblée, celui-ci me confia que le concours de son Musée constituerait un geste d'amitié et qu'il faudrait que notre projet soit à la hauteur de l'importance des relations franco-québécoises » (1).

Si le principe de la collaboration a été rapidement accepté, nous avons toutefois mis quelques temps à démarrer le projet final. En effet, un premier projet autour de «Henri IV et les arts » a d'abord été esquissé et ce, dans une perspective de présentation autant au Louvre qu'à Québec (2). C'est avec Geneviève Bresc-Bautier, conservateur général au département des Sculptures, à qui a été confié ce projet, que nous avons dès le départ commencé à travailler. Pour toutes sortes de raisons, ce projet a dû être abandonné. Nous nous sommes alors réorientés sur un projet réalisé en exclusivité pour Québec, ce qui facilitait grandement le travail.

Après la tentative « Henri IV et les arts ", une idée fait rapidement son chemin : concevoir une thématique fédératrice permettant de tirer parti de l'ensemble des huit départements du Louvre afin d'offrir une vue large de ses collections, souvent associées exclusivement à la peinture ${ }^{(3)}$. Pour ce faire, nous devions constituer un réseau de collaborateurs au Louvre et également nous appuyer sur les forces de la pratique du MNBAQ en exposition.

Revenons sur chacun de ces aspects de la démarche. D’abord, le concept. Ayant été formée au musée de la Civilisation - j’ai été membre de l'équipe fondatrice sous la direction éclairée de Roland Arpin - l'approche thématique m'était plus que familière et le déclencheur de ma réflexion a été le livre d'Adrien Goetz, Le Louvre. Les arts face à face, remis par mon collègue Yves Lacasse, directeur des collections du MNBAQ ${ }^{(4)}$. Mon expérience concrète des expositions qui supposent la mise en relation d'objets dans l'espace, et pas seulement d'images sur le papier, m'a tout de suite incitée à combiner le regard large et généreux de l'approche thématique à la dimension réaliste d'une liste d'œuvres cohérente pouvant voyager jusqu'au Québec. Rappelons que les œuvres du Louvre sont constamment sollicitées par d'innombrables prêteurs à travers le monde et que certains départements ont un très fort pourcentage de leur collection en exposition permanente. De plus, ce projet se déroulait simultanément à la collaboration du Louvre avec Atlanta, au moment aussi où l'on planifiait le Louvre à Lens et, parallèlement à l'annonce du Louvre à Abou Dhabi. Quoique notre projet reposait sur une collaboration de musée à musée, et non sur un partenariat financier, nous étions parfaitement conscients de la pression énorme qui pesait sur les collections du Louvre.

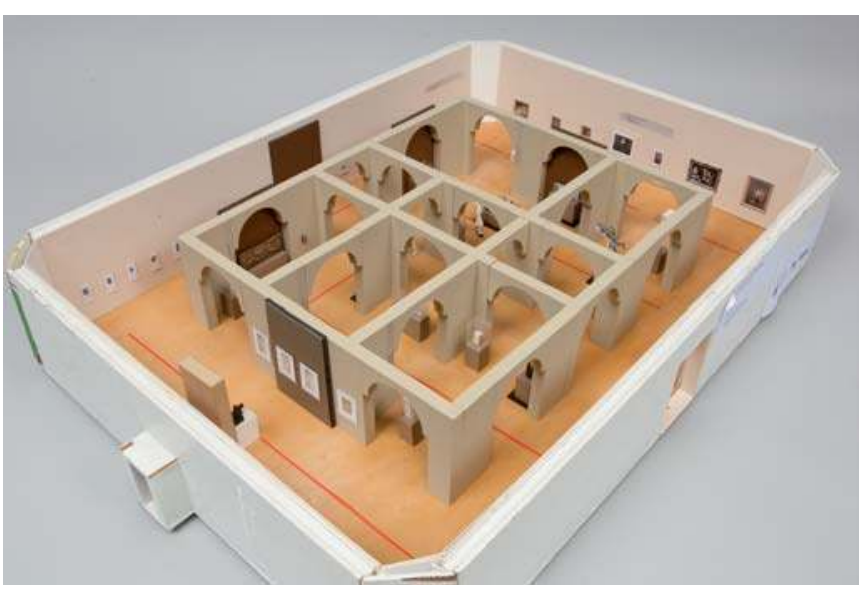

Maquette de la salle 4 présentant les thèmes «Aimer et mourir » (au centre) et «Apprendre et œuvrer » (sur les pourtours de la salle). Design : Denis Allison. (c) MNBAQ/Patrick Altman, Jean-Guy Kérouac

J'avais aussi la conviction que ce que le visiteur peut rattacher à son expérience personnelle lui permet d'appréhender immédiatement le sens de l'objet face à lui, et ce, malgré la distance des siècles ou du lieu géographique qui l'en sépare. Cette phrase d'Eugene Rochberg-Halton, dans The Meaning of Things : Domestic Symbols and the Self (1981), résume bien l'importance du sens « personnel » pour le visiteur : "Objects in exhibition display can elicit emotional responses [...] it is the emotional overlay we place upon impersonal objects that transforms them into objects of meaning » ${ }^{(5)}$. Ce courant de pensée en muséologie a été particulièrement mis à profit par des institutions telles que Parcs Canada et le musée de la Civilisation à Québec (6).

Comme c'est souvent le cas, quand tous les éléments sont en place, des associations se créent sur un mode apparemment spontané. C'est ainsi qu'au matin d'une mission à Paris en 2004, le fil conducteur de la place de l'art dans la vie de l'homme pour les civilisations représentées dans les collections du Louvre m'est apparu comme porteur de sens pour nos visiteurs. La façon dont il serait décliné a coulé de source : «Aimer et mourir », «Apprendre et œuvrer », « Habiter et embellir », « Célébrer et se divertir ». Tous ces aspects de l'activité humaine sont en effet universels et permettaient de constituer des « dossiers » pour chacun des huit départements.

Il a fallu ensuite se livrer à une recherche exhaustive dans les bases de données du Louvre pour arriver à bâtir des dossiers cohérents ne touchant qu'un département à la fois sur un sujet précis, à une période donnée, dans une discipline artistique 
unique. Par exemple, dans «Aimer et mourir » en Europe du XIVe au XIXe siècle, l'amour, un thème mythologique est décliné en sculpture avec des œuvres comme Zéphyr et Psyché (Henri Joseph Ruxthiel, Salon 1814, marbre) et Daphnis et Chloé (Jean-Pierre Cortot, Salon 1827, marbre).

Comment par la suite s'assurer de la collaboration des départements du Louvre? Nous avons eu la chance d'obtenir dès le départ le concours de Geneviève Bresc-Bautier, conservateur général du département des Sculptures, qui a tout de suite reconnu dans la thématique la « manière québécoise » et qui s'est généreusement prêtée au jeu de la marier aux merveilleuses collections du Louvre. Grâce à l'expérience et au vaste réseau de sympathie de Geneviève Bresc-Bautier, nous avons pu obtenir la collaboration de tous les départements, qui ont délégué chacun l'un de leurs conservateur ${ }^{(7)}$. Il a d'abord fallu vaincre les réticences liées à ce type de projet plus thématique mais aussi liées à la tâche supplémentaire demandée à chacun dont l'emploi du temps était déjà extrêmement chargé. Les conservateurs ont brillamment relevé le défi que représentait ce projet «à la québécoise » et contribué au contenu scientifique de l'exposition et du catalogue.

Nous désirions finalement mettre en valeur les forces de la pratique des expositions au MNBAQ. Avant d'aborder le point de vue muséologique proprement dit, je veux ici m'attarder sur les principes de gestion sur lesquels s'appuie cette pratique. On oublie trop souvent que, malgré le meilleur concept et les meilleures intentions, si les expositions ne sont pas bien gérées, on court à la catastrophe. Celles-ci sont la plupart du temps évitées de justesse à grands frais, tant sur le plan financier que sur le plan humain. Ces principes de gestion ont pour simple but de créer des conditions optimales pour le projet. Celles-ci tiennent à trois composantes : les personnes, le temps et les ressources financières. D'abord les personnes : identifier les responsables, définir les mandats de chacun et assurer un accompagnement constant pour prévenir les écueils, résoudre les problèmes, maintenir la synergie. Le temps : établir un échéancier offrant des délais suffisants pour chacune des phases clés du projet et s'assurer que les décisions pertinentes soient prises à chacune des étapes pour éviter des retours en arrière et des remises en question à la dernière minute (mentionnons que cette exposition a nécessité quatre années de travail). Finalement, prévoir des enveloppes budgétaires qui soient à la mesure des défis et contraintes du projet

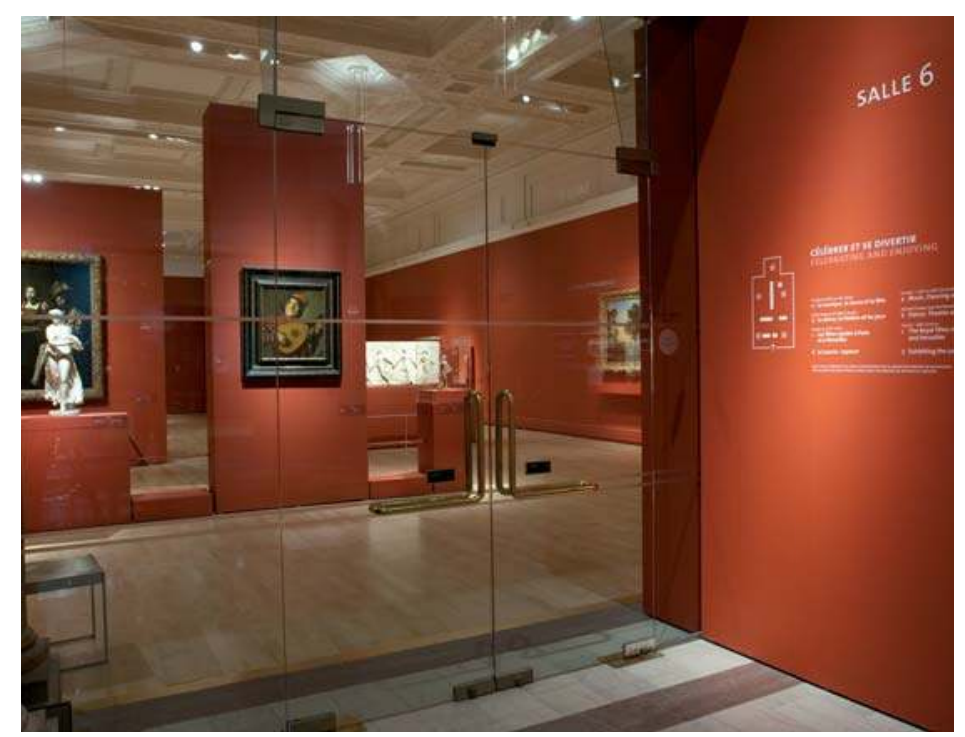

À chaque entrée de salle, un plan indiquait l'emplacement des thématiques, comme ici, à l'entrée de la salle "Célébrer et se divertir». ๑ $M N B A Q /$ Patrick Altman, Jean-Guy Kérouac

et mettre à jour les prévisions, à chaque étape clé, afin d'éviter les mauvaises surprises et les dépassements majeurs au dernier moment. Un ABC de la gestion, certes, mais combien de fois négligé. Dans le cas de l'exposition Le Louvre à Québec. Les arts et la vie, nous avons estimé qu'être exemplaire de ce point de vue contribuerait à la réalisation d'un projet exemplaire.

\section{Le meilleur des deux mondes}

En quoi ce projet s'écarte-t-il de la pratique habituelle de chacun des musées? Pour le MNBAQ, il va sans dire que travailler avec l'une des plus grandes collections au monde, avoir accès à un savoir scientifique exceptionnel par l'intermédiaire des conservateurs délégués de chacun des départements constituait une première et une occasion unique. Embrasser un thème avec une telle ambition n'aurait d'ailleurs pu être envisagé dans un autre contexte. Il y avait aussi un défi lié à la taille titanesque de notre partenaire (le MNBAQ ne compte que 125 employés) avec ses multiples départements, son histoire, sa logique interne, ses personnes clés. Un monde en soi, que nous avons découvert peu à peu avec une fascination constante, un désir de comprendre, de créer des liens, de faire avancer notre projet commun. De ce point de vue, nous avons dû faire preuve de patience et nos collègues français, de leur côté, ont dû montrer beaucoup d'ouverture pour que, quelque part, nous puissions nous rejoindre. 
En dernier lieu, mais cela du début de la démarche jusqu'à la toute fin de l'exposition, il s'agissait non seulement d'offrir une réalisation de qualité, dans la lignée des expositions habituelles du MNBAQ ${ }^{(8)}$, mais encore de répondre à des attentes à la hauteur de notre partenaire : le Louvre, musée mythique s'il en est un, lieu rêvé mais jamais visité pour nombre de Québécois. En d'autres termes, cette exposition devait attirer un très vaste public, bien au-delà de notre clientèle habituelle, et cet objectif nous a habités dès le départ ${ }^{(9)}$.

Au-delà de ces défis particuliers, nous avons été guidés par les principes propres à la pratique des expositions au MNBAQ depuis la création de la direction des expositions en 1999 (10). D'abord, la reconnaissance de la nécessité d'une vision plurielle de l'exposition qui allie l'aspect scientifique (centré sur le contenu et les œuvres), la communication (centrée sur le public) et le design (centré sur l'espace). Sans l'une ou l'autre de ces composantes, l'exposition ne saurait exister. Qu'entend-on ici par ces trois aspects? La démarche scientifique suppose une recherche originale qui permette de faire ressortir des faits nouveaux ou de créer des relations pertinentes entre des faits, des œuvres. Cette recherche originale s'exprime autant dans le catalogue que dans l'exposition, deux médiums ayant chacun leur spécificité et devant agir de façon complémentaire. Dans le cas de l'exposition, c'est l'objet, l'œuvre, qui supporte avant tout le discours. Il joue en quelque sorte le rôle principal, d'où l'importance de la cohérence entre le discours scientifique et la sélection des œuvres. Si bien que nous articulons cette liste d'œuvres sous forme de scénario pour découper les divers « arguments ». C'est ici qu'intervient la communication afin que les résultats de la recherche soient présentés aux visiteurs de la façon la plus claire et pertinente possible, selon un rythme et des temps forts calculés de manière à maintenir leur attention. Plus concrètement, cette approche se traduit dans l'exposition par des séquences spécifiques d'œuvres et par un appareil didactique efficace et discret (titres, textes, cartels, cartels commentés, documents, livret de visite, audioguide, plus rarement matériel audiovisuel, bornes informatiques) sans lequel l'exposition ne saurait remplir son rôle puisqu'elle est destinée au grand public (11).

Le design, quant à lui, part de la spécificité de l'exposition en tant que médium, laquelle prend place dans un espace où évoluent des visiteurs. Cette caractéristique du médium est non seulement pleinement assumée - toutes nos expositions font l'objet d'un travail en maquette auquel collaborent le designer et le conservateur (12) - mais elle détermine aussi le mode d'apprentissage des visiteurs du musée : «selfpaced, self-directed, non-linear, visually oriented » (13). Ici encore, le rôle du designer consiste à offrir la lecture la plus pertinente possible des ensembles d'œuvres en créant une expérience esthétique, émotionnelle et intellectuelle pour le visiteur. Cette lecture et les partis pris qui en découlent doivent servir l'œuvre et le propos et ne jamais les masquer ou les détourner. L'espace est une donnée si fondamentale de l'élaboration d'une exposition que de nombreux « ratages » sont liés à l'inadéquation

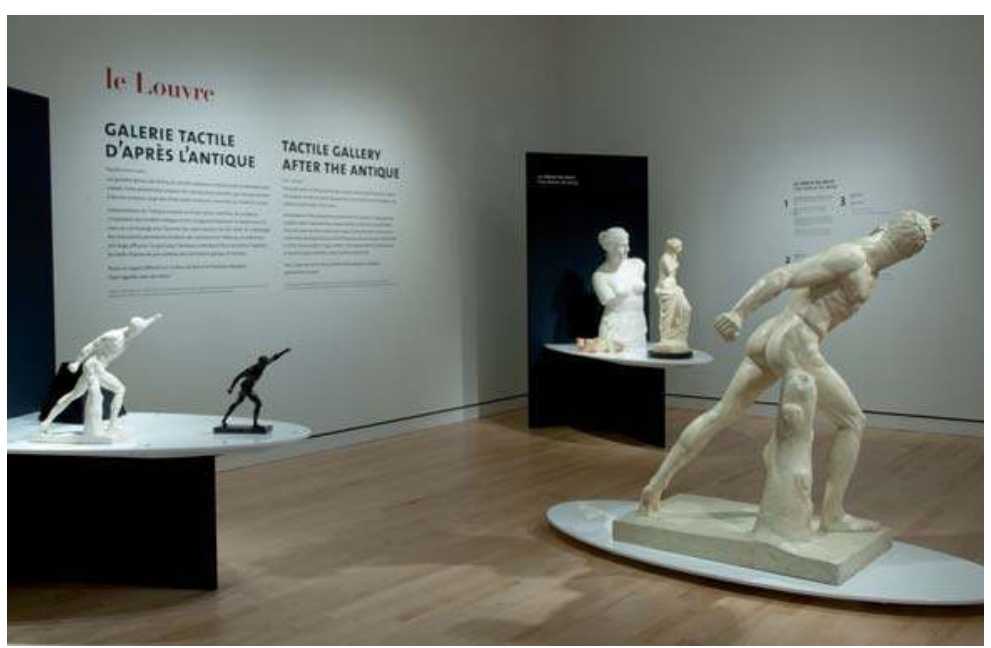

Une salle didactique pour le grand public et les mal-voyants sur le thème de la copie de l'Antique (c) MNBAQ/Patrick Altman, Jean-Guy Kérouac

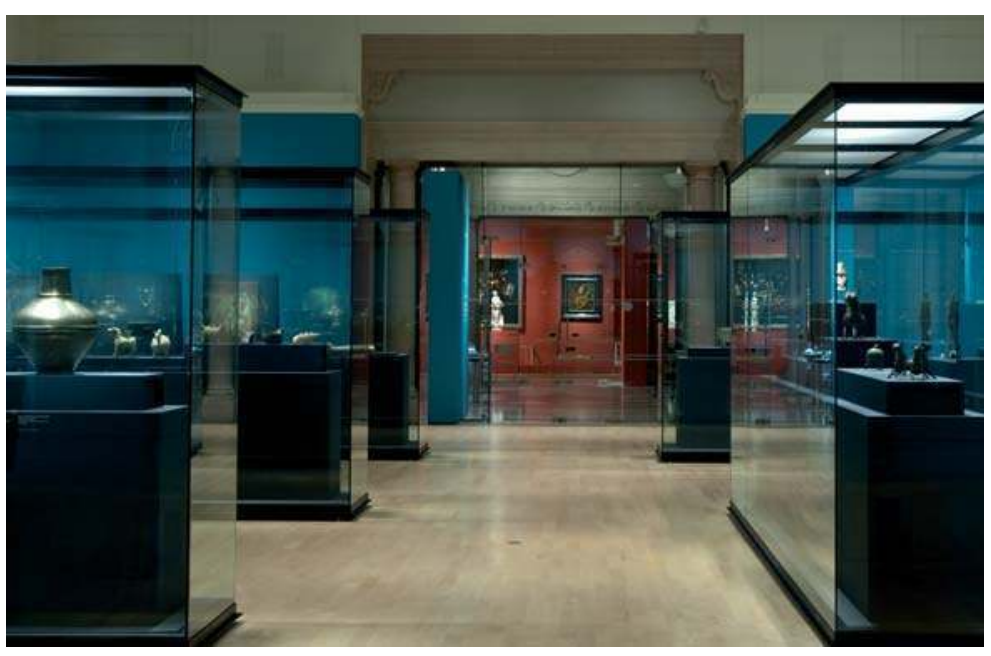

La salle " Habiter et embellir » ( ) MNBAQ/Patrick Altman, Jean-Guy Kérouac 
entre les surfaces et les types de salles et l'envergure et le type d'exposition elle-même. La mise en espace organise, met en relation, qualifie tant et si bien que les œuvres semblent se révéler sous un tout autre jour, non seulement pour le grand public mais aussi, à plus forte raison, aux yeux des concepteurs de l'exposition et, au premier chef, du conservateur.

Du côté du musée du Louvre, en quoi cette exposition différait-elle de sa pratique ? Voici ce que répond Henri Loyrette dans sa préface au catalogue : «Ce n'était pas un sujet d'exposition classique pour le Louvre, où en général on étudie un artiste, une époque, une civilisation, un style ou une technique. Bouleversant leurs habitudes mentales, les conservateurs se sont mis à l'école de la muséologie canadienne. Ils ont joué le jeu d'une quête originale des objets, dessins, sculptures ou tableaux les plus démonstratifs - et les plus beaux - pour donner un panorama structuré de ce que fut la place de l'art dans la vie traditionnelle des civilisations couvertes par le Louvre » (14). Ainsi l'approche thématique et la lecture transversale des collections apportaient à nos collègues du Louvre une nouvelle expérience. Il s'agissait pour le Louvre d'une expérience ponctuelle d'autant plus acceptable qu'elle ne se retrouvait pas en leurs murs mais en sol québécois, là où cette approche muséologique est bien enracinée. C'est du côté du Louvre-Lens que cette expérience a eu une plus grande résonance, les élus de la région ainsi que certains membres de l'équipe du Louvre se sont déplacés pour voir l'exposition à Québec. Depuis, nous entretenons des échanges Louvre-Québec sur la muséographie et la médiation par le biais de rencontres et d'ateliers (15).

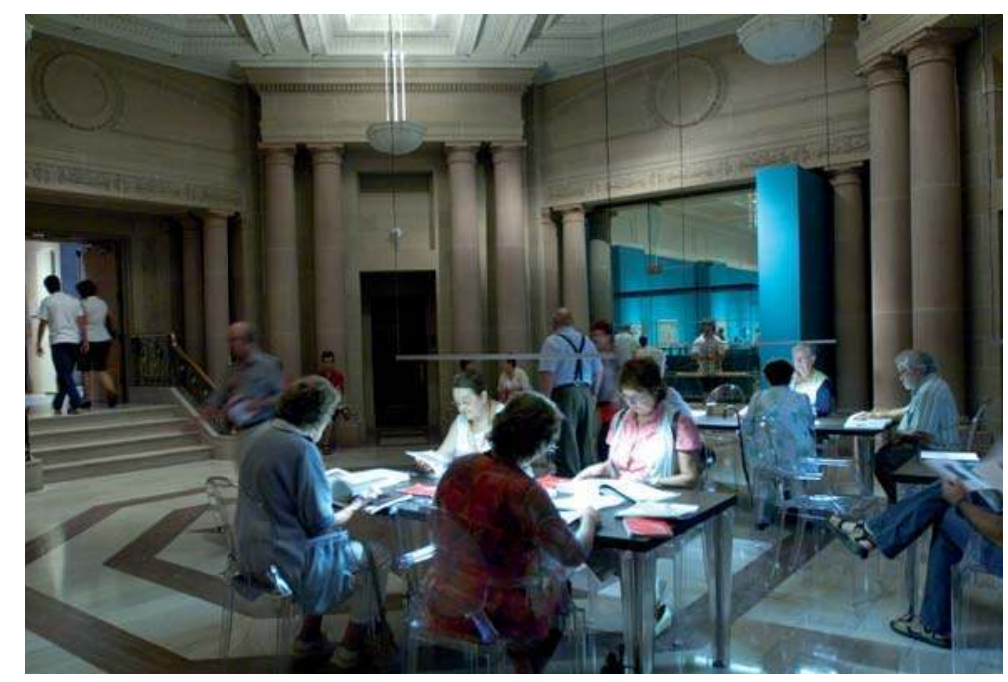

Dans la rotonde reliant les salles 5 et 6 , une salle de consultation du catalogue qui tenait également lieu d'espace de repos. ๑) MNBAQ/Parrick Altman, Jean-Guy Kérouac

\section{Les résultats}

Une exposition déployée sur $1200 \mathrm{~m}^{2}$, avec 276 œuvres des huit départements déclinées en 23 dossiers thématiques (16) regroupés sous quatre grandes rubriques : «Aimer et mourir », «Apprendre et œuvrer », « Habiter et embellir », « Célébrer et se divertir ». Un catalogue de 287 pages (en coédition avec Hazan) émaillé de 274 illustrations auquel ont contribué 15 auteurs, une fréquentation de 464576 visiteurs, un taux de satisfaction moyen de $8,7 / 10^{(17)}$, un comportement exemplaire de visite avec des foules patientes, studieuses, concentrées. La presse locale a été globalement enthousiaste : "Le Louvre à Québec. Exceptionnel " (Media Matin Québec), "Le Louvre à couper le souffle » (Le Soleil), "Le Louvre comme vous ne l'avez jamais vu! " (La Presse). Le Globe and Mail, journal pancanadien, soulignait que l'exposition Le Louvre à Québec. Les arts et la vie était un « highlight » des célébrations du 400 e anniversaire de la ville de Québec. Quant à nos collègues du Louvre, ils ont apprécié l'expérience et le résultat en salle, plus particulièrement l'espace généreux accordé aux œuvres qui permettait de diriger le regard et de mettre en valeur des pièces qui, au Louvre, cohabitent dans des ensembles beaucoup plus denses.

Comment expliquer ce succès ? Certes, le pouvoir d'attraction du Louvre est indéniable et il a exercé son effet dès la première heure, si bien que notre plus grande crainte a toujours été, face à des attentes très élevées, de décevoir. Et, sans l'ombre d'un doute, nous avons déçu : ceux pour qui seule l'approche "classique » est justifiée en exposition, ceux pour qui le Louvre n'est que son département des Peintures, ceux pour qui seuls les « chefs-d'œuvre » méritent de représenter ce musée. Or nous avons écarté d'emblée l'approche « chefs-d'œuvre » sachant qu'il était peine perdue de construire du sens uniquement de cette façon. Nous avons trouvé plus judicieux de refléter la variété des disciplines artistiques, des périodes et des civilisations auxquelles sont consacrées les collections du Louvre en regroupant le tout de façon thématique.

Par ailleurs, nous avons voulu, quoique modestement, nous inspirer de l'expérience muséographique offerte par le Louvre : l'une des salles évoquait les cours de sculptures et les galeries latérales de peintures, une autre figurait une enfilade de vitrines d'objets, une dernière arborait une couleur récurrente dans les grandes salles de peintures : le rouge. 
Nous avions aussi parfaitement conscience de la difficulté que pouvait représenter pour le visiteur la juste appréciation de cette multitude d'œuvres de nature, de provenance et de périodes très diverses. Il fallait de plus tenir compte de la grande affluence qui, en général, gêne la lecture des textes et des cartels. Pour toutes ces raisons, nous avons opté pour un carnet de visite très complet incluant des textes des grandes sections, des dossiers et tous les cartels et commentaires qui les accompagnaient. Avec ses 176 pages, il constituait un outil, non pas à lire exhaustivement mais plutôt à consulter au gré des intérêts de chacun et, comme les goûts sont infiniment variés, nous y avons consigné l'information sur l'ensemble des dossiers et des œuvres. Un parti pris qui n'aurait pu tenir si le matériel didactique avait été placé aux murs et qui a conditionné les modalités de visite au-delà de nos attentes. En effet, les visiteurs en possession de cette "mine " d'information (un carnet maniable, très simple à consulter, avec des textes courts mais fouillés) pouvaient se déplacer de façon autonome, créant leur propre parcours ponctué de pauses, de moments de lecture, guide à la main, faisant cercle autour des œuvres ou les observant à distance.

L'équipe du MNBAQ est consciente du fait qu'il s'agit d'une expérience unique que nous avons vécue et partagée avec nos visiteurs. Une expérience qui nous a permis de mettre à l'épreuve notre pratique de l'exposition et de bénéficier de l'extraordinaire «magie » du Louvre, de son équipe, de sa collection, de son histoire, tout en y ajoutant, avec le plus grand respect, une petite touche bien québécoise.

\section{Notes}

(1) Une grande rencontre muséale à Québec, préface de John R. Porter dans Line Ouellet et al. Le Louvre à Québec. Les arts et la vie. Québec : musée national des Beaux-Arts du Québec ; Paris : Hazan, musée du Louvre, 2008.

(2) Une petite section avant-propos de l'exposition Le Louvre à Québec. Les arts et la vie fait d'ailleurs un clin d'œil à Henri IV, le roi régnant au moment de la fondation de la ville de Québec.

(3) Antiquités égyptiennes, Antiquités grecques, romaines et étrusques, Antiquités orientales, Arts de l'Islam, Arts graphiques, Objets d'art, Peintures, Sculptures.
(4) Goetz, A. Le Louvre. Les arts face à face. Paris : Hazan, 2007, 280 p.

(5) Mihaly Csikszentmihalyi et Eugene Rochberg-Halton The Meaning of Things : Domestic Symbols and the Self. New York : Cambridge University Press, 1981.

(6) Sur l'histoire de la muséologie au Canada, voir Ouellet, L. La muséographie au Canada : une pratique réputée, une formation en devenir, Perspective, $\mathrm{n}^{\circ} 3$, 2008, pp. 513-526.

(7) Ces conservateurs sont : Élisabeth Antoine, conservateur, département des Objets d'art ; Agnès Benoit, conservateur en chef, département des Antiquités orientales ; Geneviève Bresc-Bautier, conservateur général, département des Sculptures ; Blaise Ducos, conservateur, département des Peintures ; Marc Étienne, conservateur, département des Antiquités égyptiennes ; Catherine Loisel, conservateur en chef, département des Arts graphiques ; Sophie Makariou, conservateur, département des Arts de l'Islam ; Jean-Luc Martinez, conservateur en chef, département des Antiquités grecques, étrusques et romaines.

(8) Le MNBAQ organise chaque année une dizaine d'expositions d'art québécois (le cœur de sa collection), d'art contemporain et d'art international. (9) Le MNBAQ (fondé en 1933) a réalisé au cours de son histoire quelques expositions ayant attiré un très vaste public, parmi lesquelles Rodin à Québec, du 4 juin au 13 septembre 1998, avec 525000 visiteurs. (10) La traditionnelle fonction de conservateur en chef a alors été partagée en deux postes : un directeur des collections et de la recherche et une directrice des expositions et des publications. Les conservateurs, responsables de secteurs de la collection, relèvent de la direction des collections et de la recherche et travaillent également pour la direction des expositions selon les mandats qui leur sont confiés; quelques conservateurs (qui n'ont pas de secteur de collection) relèvent uniquement de la direction des expositions. Ce modèle s'inspire de celui développé par la Tate. (11) Nous privilégions cette approche également pour nos expositions d'art contemporain et nos expositions permanentes.

(12) Voir à ce sujet Emmanuelle Vieira et Line Ouellet Design d'exposition. Dix mises en espace d'expositions au Musée national des beaux-arts du Québec. Québec : musée national des Beaux-Arts du Québec, 2004, $136 \mathrm{p}$.

(13) Screven Chandler Museum learning and the casual visitor. 1987.

(14) Une expérience innovante, préface d'Henri Loyrette dans Line Ouellet et al. Le Louvre à Québec. Les arts et la vie. Québec : musée national des Beaux-Arts du Québec ; Paris : Hazan, musée du Louvre, 2008.

(15) Un premier atelier a eu lieu à Montréal et à Québec en octobre 2009, avant la fin de l'exposition, et un second atelier aura lieu en France en 2010.

(16) Rédigés par Pierre Landry qui, avec le designer Denis Allison et le coordonnateur aux opérations André Sylvain, a grandement contribué à ce projet d'envergure.

(17) Sondage réalisé auprès des visiteurs de l'exposition Le Louvre à Québec. Les arts et la vie, Léger Marketing pour le MNBAQ, novembre 2008, 23 p 